\title{
Interactive comment on "Contributions of different anthropogenic volatile organic compound sources to ozone formation at a receptor site in the Pearl River Delta region and its policy implications" by
}

\section{Z. He et al.}

\section{Anonymous Referee \#1}

Received and published: 8 March 2019

The authors presented hourly resolved measurements of volatile organic compounds (VOCs) at a receptor site in the Pearl River Delta (PRD) region in China. A receptor model, positive matrix factorization (PMF), was used to apportion sources of VOCs, by taking into account of photochemical degradation of some VOCs. Results showed that four sources, including gasoline vehicular emission, diesel vehicular emission, biomass burning, and solvent usage, are major contributors to anthropogenic VOCs at the site. A photochemical box model with the master chemical mechanism was also used to evaluate the contributions of those VOCs to ozone formation, with vehicular emission 
found to be the most significant. Further analysis to test abatement scenarios was also performed for policy implication.

Overall, this is a nice data set with thorough analysis. The manuscript is generally well written and is surely of interest to readers of ACP. I therefore recommend minor revision before publication, with below comments for the authors.

Interactive

\section{Major:}

1. I do not understand that in Section 3.3, why did the authors sum up gasoline and diesel vehicular emissions in their discussion on the contributions of different VOC sources to ozone formation. It is of importance for policy making, as to which type of vehicular to control (e.g., with priority), if we have more detailed understanding on whether vehicles run on gasoline or diesel have more potential in VOC emission that is related to ozone formation. Can the authors justify and clarify?

2. Using the same set of data, the same group of authors published in Journal of Environmental Science (JES) recently. Although the foci of the two papers are different, with the JES paper on isoprene and their oxidation products and this one on anthropogenic VOCs, I do like to see some connection between the two papers as they are based on the same data set (it was not even cited here). More importantly, what is the similarity and difference in methodology between these two papers? Would there be any bias if the whole chunk of biogenic VOCs were taken out from photochemical box model (e.g., the source/fate of $\mathrm{OH}$ radicals and ozone)?

3. Page 12, line 19-25. I do not understand the assertion here that correlation will be distorted if two VOCs from the same source have different photochemical reaction rates. The authors used the photochemical age concept in the paragraph right after it, which means that correction can still be retained if the two VOCs react in a proportional manner with $\mathrm{OH}$ radicals (and assuming no other fates). The rationale of these two paragraphs seems contradicting. Please clarify. 
4. P7/L37: I would strongly suggest the authors include more details on why a fourfactor solution was chosen. Diagnostic analysis by comparing three-factor and fivefactor solutions would be useful, even as supplementary materials. "a good fit to the data and the most meaningful results" is just too descriptive and not very convincing.

Minor:

1. P2/L41: "complex, nonlinear" to "complex and nonlinear".

2. P2/L44: "VOCs and NOx limited" to "VOC- and NOx-limited"; "VOCs-limited" to "VOC-limited", and in other places as well.

3. P3/L60: "emission-inventory" to "emission inventory".

4. P4/L84: did Ling et al., JES, 2019 take photochemical processing into account?

5. P6/L19: remove "," after "contributions".

6. P7/L32: "the detection limit" to "their detection limits".

7. P7/L35: why MTBE and ACN so special and not considered as "VOCs"?

8. P8/L58: add "divided by" after "production"?

9. P9/L89\&L91: "that" to "those".

10. P9/L94-96: how can variations of VOCs suggest photochemical processing? It could be just variations on sources. Please clarify.

11. P9/L96: delete "to be".

12. P12/L34-35: these rate constants appeared later in the next page and look redundant. Please remove.

13. P13/Eq 6: k_VOC instead of k_NMHC? It would be good to have a table showing the rate constants for each VOCs and appropriate citation.

14. P14/L60\&L62: "reaction rate" should be "reaction rate constant"? 
15. P14/L71: add "the" before "rest species".

16. P15/L89: remove "," after "VOCs".

17. P15/L93: "acetonitrile" to "ACN" (you defined it early).

18. P15/L00: "peak" or "valley"?

19. P17/L22: "relative" to "relatively".

20. P18/L29: add "a" before "more".

21. P23/L31: remove "cluster".

22. P24/L44-P25/L65: I would suggest to shorten this paragraph to a few sentences to make the point: although there are some control measures on VOC emission from vehicles, there is limited control on biomass burning etc.

23. Figure 4: can the authors use another panel to show ratios too?

24. Figures in general: it would be more reader friendly if the authors can use a bigger font size for most of the figures.

Interactive comment on Atmos. Chem. Phys. Discuss., https://doi.org/10.5194/acp-2018-1293, 2019. 